\title{
LA EDUCACIÓN FÍSICA Y LAS RELACIONES SOCIALES EN EDUCACIÓN PRIMARIA
}

\author{
Tania Carbonell Ventura \\ Estudiante en Máster de Dificultades del Aprendizaje y Trastornos del Lenguaje en la UniversitatOberta de \\ Catalunya \\ taniacarbonellven@gmail.com \\ José L. Antoñanzas Laborda \\ Profesor de la Facultad de Educación, \\ Universidad de Zaragoza \\ Ángela Lope Álvarez \\ Profesora de la Facultad de Educación, \\ Universidad de Zaragoza
}

Fecha de Recepción: 28 Marzo 2018

Fecha de Admisión: 10 Abril 2018

\section{RESUMEN}

Este estudio llevado a cabo es una propuesta de intervención educativa basada en la Expresión Corporal, en la cual se defiende que las relaciones sociales se pueden mejorar a través de la Educación Física.

El principal objetivo de este estudio es la mejora de la desinhibición en el aula de Primaria a través del juego, concretamente del aprendizaje cooperativo, que ayudará al niño a tener mejores habilidades sociales para relacionarse con los demás. La propuesta de intervención consta de siete sesiones basadas en la Expresión Corporal, la cual presenta actividades grupales para que los alumnos puedan desinhibirse y expresarse con los demás de forma natural, así podrán mejorar su vínculo social con el grupo.

Palabras clave: expresión corporal; aprendizaje cooperativo; Educación Física; relaciones y habilidades sociales; Educación Primaria

\section{ABSTRACT}

Physical education and social relationship in primary education.

This study is a proposal of educational intervention based on corporal expression, in which the belief that social relations can be improved through Physical Education is defended.

The main objective of this study is to improve disinhibition in the Primary classroom through games, particularly cooperative learning games, which will help the child to develop better social skills in order to have a better knowledge in how to relate with others. The intervention proposal con- 


\section{LA EDUCACIÓN FÍSICA Y LAS RELACIONES SOCIALES EN EDUCACIÓN PRIMARIA}

sists of seven sessions based on corporal expression, which have group activities in which students will lose their inhibitions and express themselves in a natural manner so that student will improve their social links with the group.

Keywords: corporal expression; cooperative learning; physical education; social relationship and skills; Primary Education

\section{INTRODUCCIÓN}

La Educación Física debe convertirse en un método para que los alumnos se reconozcan e interactúen con las personas de manera sana, y a la vez adquieran destrezas y habilidades sociales, que les permitan tener buenas relaciones con su familia, amigos y en general con todo su entorno social. Mediante las actividades, juegos y ejercicios en base a la Expresión Corporal, los alumnos pueden interactuar de forma directa, expresando sentimientos y deseos, mediante su cuerpo, poniéndose de acuerdo con sus compañeros.

A pesar de que la Expresión Corporal no siempre ha formado parte de los contenidos de Educación Física, dichos contenidos han ido apareciendo de forma continuada relacionados con la espontaneidad, expresividad, sencillez y naturalidad del momento (Ortiz, 2002).

Según Learreta, Ruano y Sierra (2006) esta disciplina contribuye al desarrollo de las capacidades humanas de cada niño en relación con la expresión, la comunicación y la creación.

Al tener que experimentar esas sensaciones, se produce un contacto con el compañero, a veces resulta positivo y otras negativo, es en ese momento, donde tiene cabida la educación.

De este modo, las relaciones sociales son un pilar fundamental que tiene que tener toda persona para poder interrelacionarse con los demás.

\section{EDUCACIÓN FÍSICA}

La Educación Física tiene como propósito desarrollar en su totalidad el área fisicomotriz, higiene, social, psiclógica y moral.

Se analiza inicialmente el término Educación Física según el Real Decreto 126/2014 del currículo básico de educación primaria de Aragón: La Educación Física persigue el desarrollo de la persona en su globalidad, lo que implica aspectos motores, biológicos, cognitivos, de relación interpersonal y afectivo-emocional.

Según Perezplata (2006) la Educación Física influye en la educación a la salud y al cuerpomente.Por eso, enseña los valores necesarios para el alumno como persona social.

De igual manera, Grasten(2015) afirma que para conseguir los objetivos de esta área, debe haber motivación para participar. Si el niño disfruta de la actividad física, éste va a estar motivado para impartir las clases de educación física en su totalidad.

La Educación Física es un área curricular que favorece el desarrollo integral de los alumnos, ya sea a nivel físico, psíquico o sociológico. Se aprecia la actividad física como una excelente vía de comunicación social, la cual permite la relación con los demás. 
Figura 1. Los beneficios que tiene la Educación Física en el desarrollo del alumnado

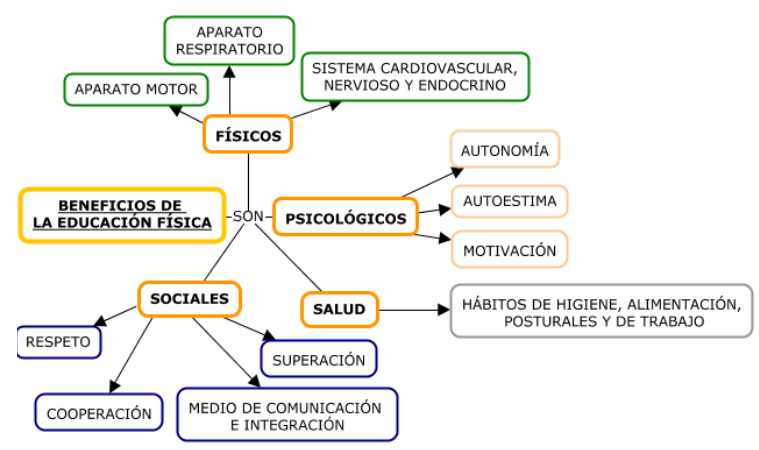

\section{DESARROLLO DEL ALUMNO EN EL ÁREA DE EDUCACIÓN FÍSICA}

A través de la Educación Física se pueden trabajar diferentes desarrollos del alumno:

\section{Desarrollo cognitivo}

Según Lagunas (2006) la Educación Física mejora la capacidad de análisis de los conceptos, desarrolla la comprensión, aumenta la memoria a corto y medio plazo, mejora la coordinación y desarrolla la imaginación.

Gracias a esto, los niños tienen un pensamiento más receptivo a la hora de desarrollar el aprendizaje.

Este área también trabaja el conocimiento requerido para ejecutar los movimientos motores complejos, por lo que hay cambios fisiológicos a largo plazo en el cerebro inducido por el ejercicio aeróbico (Carson, 2015).

Por otra parte, la inteligencia abandona su subjetividad y el egocentrismo y se convierte en lógica (Jurado, 2015).

Debido a esta evolución, las explicaciones que dan los niños cada vez son más razonadas, se centran en el porqué de las cosas.

\section{Desarrollo social}

La Educación Física afecta a nivel social porque los niños al jugar con iguales, afianzan el compañerismo y las relaciones sociales ya que les facilita la comunicación y la interacción social, asimismo también se fomenta la educación en valores, concretamente, compartir, debido a la gran cantidad de materiales que se utilizan en clase (Lagunas, 2006).

La convivencia y el compañerismo de desarrollan porque tienden a formarse grupos (Jurado, 2015), ya que en esta edad comienzan los conocimientos de las reglas y los roles sociales (Lagunas, 2006).

Además, si los niños están motivados en clase hará que incremente el sentimiento de estar con los amigos con una actitud positiva (Dos Santos, 2015).

\section{Desarrollo afectivo}

El deporte ayuda a eliminar los miedos, sobre todo con los contenidos de teatro y dramatiza- 


\section{LA EDUCACIÓN FÍSICA Y LAS RELACIONES SOCIALES EN EDUCACIÓN PRIMARIA}

ción, lo que conlleva a aprender a ponerse en el lugar de los demás. También, aumenta la confianza en sí mismo y su autoestima (Lagunas, 2006), igualmente si el maestro confía en sí mismo y en el niño, éste percibirá dicha confianza y confiará en él mismo, por ello el maestro tiene que crear un clima seguro para que los niños se sientan a gusto (Velea, 2013).

El proceso de socialización viene marcado por dos grandes influencias: profesorado y amigos (Jurado, 2015).

Dicho proceso se consigue porque los niños empiezan a pensar de manera más autónoma y poco a poco son más autosuficientes.

\section{Desarrollo motor}

Tal como dice Antunes (2015) con la edad se va desarrollando la coordinación motora. Se desarrolla el control postural y respiratorio, y nos encontramos en una edad con la lateralidad afianzada (Lagunas, 2006).

El área de Educación Física debe proporcionar a los alumnos la adquisición de unos patrones motrices básicos que vayan de lo más global a lo más específico.Los autores citados anteriormente opinan similar en base a que esta área desarrolla tanto la socialización, como las capacidades motoras básicas y coordinativas, también contribuye a la formación de la personalidad de los alumnos, y por supuesto, fomenta la educación en valores a través del deporte.

\section{PROCESOS COGNITIVOS}

Trost (2009) afirma que en la etapa escolar la actividad física está asociada con el rendimiento académico y el comportamiento en el aula deseable. Por ello, muchos de los procesos cognitivos que se observan en el alumnado, y que influyen directamente en el rendimiento académico, son: el auto-concepto, la autoestima, la motivación (extrínseca e intrínseca) y las actitudes prosociales.

\section{Auto-concepto y autoestima}

El auto-concepto y la autoestima personal son dos pensamientos que se instalan en nuestra conciencia y aunque parezcan dos conceptos parecidos, tienen diferencias notorias.

Según Cassá (2007), la autoestima es una manera de evaluarnos a nosotros mismos, es decir, la imagen que tiene una persona de sí misma (auto-concepto), es un paso fundamental para el desarrollo de la autoestima. Por la simple razón que cuando nos conocemos, tenemos que aprender a aceptarnos (autoestima).

Por ello, es importante trabajar dichos procesos en el área de Educación Física, de tal manera que los niños crezcan con un sentimiento positivo de sí mismos. Las experiencias de éxito influyen en la eficacia de hacer las cosas, por ello la confianza en nosotros mismos nos prepara para abordar cosas nuevas.

Parque (2008) dice que una de sus investigaciones ha demostrado que haciendo movimientos deportivos mejora el auto-concepto, la soledad, el estrés y la depresión.

Partiendo de esta investigación, también se puede mejorar el auto-concepto de sí mismo a través del juego cooperativo como dicen Box y Little (2003) quienes examinaron los efectos del aprendizaje cooperativo en el auto-concepto y llegaron a la conclusión de que el auto-concepto de los estudiantes es influenciado positivamente por las prácticas de aprendizaje cooperativo.

Por lo tanto, desarrollar positivamente la autoestima y el auto-concepto a través de la cooperación es importante, ya que los juegos cooperativos suponen un desafío constante para el grupo y proporcionan el momento de tomar decisiones importantes donde el error no es sancionado, de esta manera todos los alumnos son respetados dentro de una actividad que favorece la formación de un auto-concepto positivo. De la misma manera, al tomar sus propias decisiones y no estar influidos 
por elementos externos, desarrollan autoconfianza en sí mismos, por lo tanto favorece a la autoestima del alumno.

\section{Motivación}

Ajello (2003) señala que la motivación tiene que ser entendida como la trama que sostiene el desarrollo de aquellas actividades que son significativas para la persona y en las queesta toma parte.

La motivación puede reflejarse por diversas causas como: naturaleza de la tarea, estructuración y organización de la tarea por el profesor... en definitiva la motivación en el alumno para un buen trabajo viene de la mano gracias a las expectativas que se depositan en él por sus compañeros y el propio docente, ya que un alumno que no se integra en el grupo, que no se siente respaldado por sus compañeros nunca podrá motivarse con la tarea propuesta ni tampoco la afrontará con éxito (Lorenzana, 2012).

Los objetivos propuestos por el profesor al comenzar cualquier unidad didáctica se tratan de metas de tipo extrínsecas, es decir, si los alumnos tienen una motivación externa ellos se sentirán motivados para alcanzar los objetivos que propone el profesor con éxito. Sin embargo, si progresivamente, este trabajo va obteniendo resultados positivos, y los propios alumnos son conscientes de que su aprendizaje se está enriqueciendo, entonces la motivación pasará a ser intrínseca, el alumno pasará de esforzarse por una recompensa a trabajar constantemente por su propio desarrollo personal, físico e intelectual (Lorenzana, 2012).

Por lo tanto, los estudiantes que tienen una actitud positiva hacia las actividades físicas en las clases de educación física (Cardinales y Cardinales, 2001), o que indican que están más motivados o que se divierten más en estas clases (Cox, Smith y Williamns, 2008), tienen una mayor probabilidad de ser activo fuera del contexto educativo.

Por otro lado, tal como dice Bamburg (1994) las expectativas del maestro son uno de los factores que determinan qué tan bien y qué tanto aprenden los estudiantes.

La motivación generada por el maestro es un factor determinante en la percepción de la utilidad y la importancia de la Educación Física de los estudiantes (Moreno y Llamas, 2007).

La comprensión por parte del maestro del proceso motivacional y su importancia en las clases de educación física, constituye un conocimiento imprescindible para lograr un comportamiento favorable de los alumnos hacia las clases.

Asimismo si el maestro estructura un ambiente de apoyo a la autonomía, los alumnos darán alternativas y actividades diseñadas a promover la relación social (Bryan y Solmn, 2007).

Además de la importancia que tiene el maestro, también son primordiales los factores sociales tales como la promoción del aprendizaje cooperativo, el énfasis en el mejoramiento personal, y la selección de las tareas pueden llevar a resultados motivacionales positivos hacia la Educación Física (Ntoumanis, 2001).

Dicho esto, la Educación física desempeña un papel muy importante en la adquisición de un comportamiento saludable de los estudiantes (Moreno y Llamas, 2007; Nuviala, Gómez-López. Pérez, y Nuviala, 2011).

\section{Actitudes prosociales}

Un factor muy importante en las relaciones sociales del alumnado que los maestros debemos tener presente es la psicología de los alumnos ya que desempeña un papel fundamental en su educación.

La conducta prosocial ha sido definida como "toda conducta social positiva que se realiza para beneficiar a otro con/sin motivación altruista incluyendo conductas como dar, ayudar, cooperar, compartir, consolar..." (Garaigordobil, 2003, pp. 100). 


\section{LA EDUCACIÓN FÍSICA Y LAS RELACIONES SOCIALES EN EDUCACIÓN PRIMARIA}

En Educación Física se destacan características únicas en cuanto a la interrelación que se produce entre los alumnos, lo que sin duda constituye un ambiente idóneo para el fomento de actitudes y valores prosociales de los estudiantes (Holt, Sehn, Spence, Amanda yBall, 2012). Sin dejar de lado que las creencias, pensamientos, juicios y sentimientos que tenemos hacia los alumnos influirán notablemente en el comportamiento de trabajo del grupo, por eso es importante creer en nuestros alumnos (Bamburg y Jerry, 1994). James Raffini (1993) afirma que cuando los maestros creen en sus alumnos, éstos creen también en sí mismos.

Las relaciones sociales hay que trabajarlas desde que son pequeños, de esta manera tendrán un aprendizaje basado en valores y habilidades sociales cuando estén en el proceso de formalizar su personalidad.

\section{ESTILOS DE ENSEÑANZA}

Según Delgado (1989) dice que los estilos de enseñanza son un modo o forma que adoptan las relaciones didácticas entre los elementos personales del proceso de enseñanza-aprendizaje tanto a nivel técnico y comunicativo, como a nivel de organización del grupo-clase y sus relaciones afectivas en función de las decisiones que tome el profesor.

Según la clasificación de Sicilia Camacho y Delgado Noguera (2002) existen estos tipos de estilos de enseñanza que un profesor puede tomar en sus clases:

Tabla 1. Palabras clave en los estilos de enseñanza

\begin{tabular}{|l|l|}
\hline ESTILOS DE ENSEÑANZA & PALABRA CLAVE \\
\hline EE tradicionales & Orden, tarea \\
\hline EE fomentan la individualización & Individualización, alumnado \\
\hline EE posibilitan la participación & $\begin{array}{l}\text { Participación en técnica de enseñanza, delegación } \\
\text { de funciones }\end{array}$ \\
\hline EE propician la socialización & Grupo, cooperación, socialización \\
\hline EE implican cognoscitivamente & Resolver tareas, indagación, búsqueda \\
\hline EE favorecen la creatividad & Diversidad, pensamiento divergente, creación \\
\hline
\end{tabular}

La propuesta de intervención se va a centrar en tres estilos de enseñanza; estilo de socialización, creativos y estilos que implican cognoscitivamente. Se trata de tres estilos de enseñanza donde la cooperación está siempre presente y son los más adecuados para trabajar la socialización.

\section{Estilos de enseñanza que propician la socialización}

En esta categoría de estilos es primordial el juego de roles, la simulación social, así como el trabajo grupal y las diferentes técnicas de dinámica de grupos (Joyce y Weil, 1985). Estos estilos se focalizan en los objetivos sociales y en los contenidos actitudinales así como el respeto de normas y el desarrollo de los valores. Las palabras clave que intervienen en este estilo son grupos, socialización y cooperación. Por lo tanto, el profesor tiene que dar protagonismo al grupo y se apoya en la dinámica del mismo para plantear trabajos de tipo colectivo, donde lo importante no es la ejecución individual sino el trabajo colaborativo en la clase de Educación Física.

En conclusión, se trata de un tipo de enseñanza a través de la interacción de los alumnos mediante el intercambio de ideas, selección de roles, construcciones colaborativas y actividades grupales (Nonaka y Takeuchi, 1995).

\section{Objetivos de la enseñanza socializadora}

El objetivo general es enfrentar las estructuras mentales de las personas con las de otras para promover un refinamiento continuo sobre un contexto educativo definido (Vigotsky, 1978). 
Además, con este tipo de enseñanza se fomenta:

Conseguir que un individuo sea socialmente eficaz

Estimular el espíritu de cooperación y solidaridad

Enseñar a trabajar en equipo

Fomentar la responsabilidad en grupo

\section{Estilos de enseñanza que implican cognoscitivamente de forma más directa al alumno en su aprendizaje}

Se encuentra el descubrimiento guiado, la resolución de problemas y el planteamiento de situaciones tácticas. Se trata de una enseñanza a través de la indagación, la forma diferente de enfocar la información de la tarea, por lo que se indica qué hay que realizar pero no cómo hay que realizarlo, sino la forma de abordar la tarea.

\section{Estilos de enseñanza que favorecen la creatividad}

Como su propio nombre indica serán modalidades donde se deja libertad para la creación motriz. Por lo que cuenta con la libre exploración, la búsqueda de formas nuevas sin un objetivo necesariamente de eficacia y la actitud del profesor deben servir como estímulo y de control de contingencias.

\section{RELACIONES SOCIALES}

VaelloOrts (2005) nos habla de un concepto propio. Son el conjunto de capacidades para emitir conductas eficaces en situaciones interpersonales con la finalidad de obtener respuestas gratificantes de los demás. Este término engloba destrezas específicas aplicables a diferentes situaciones de intercambio social.

Es importante saber, que dicha personalidad necesita un trabajo diario y constante, que vaya desarrollándose poco a poco en los alumnos, y que no solo se aplique en el núcleo familiar sino también en el contexto escolar junto con la educación socioemocional. Tal como diceVaelloOrts (2005), es imprescindible ser conscientes lo que supone que cualquier momento es aprovechable para mejorar la convivencia y que cualquier comportamiento tiene influencia en el clima social del aula.

\section{COMPETENCIA SOCIAL.}

Según Trianes, Muñoz y Jiménez (2000) la competencia social es una expresión que engloba dimensiones cognitivas y afectivas positivas que se traducen en conductas congruentes valoradas por la comunidad. Estos comportamientosfavorecen la adaptación, la percepción de autoeficacia, la aceptación de los otros y los refuerzos agradables, es decir, el bienestar.

Las habilidades sociales son un rasgo esencial en las relaciones entre iguales y en la competencia social, porque dependiendo de si el niño tiene mejores o peores capacidades sociales, la relación con los demás será diferente.

Monjas (2007) constituye tres elementos importantes que forman las habilidades sociales: (a). Cognitivos (lo que piensa el niño). (b). Emocionales (lo que siente la persona); y (c) Conductuales (lo que se dice/hace). Esta autora, a su vez, defiende que las habilidades sociales son educables, considera que son conductas aprendidas que pueden cambiar utilizando tácticas adecuadas, teniendo en cuenta el contexto del niño.

La competencia social en el aula se puede trabajar partiendo de 4 conceptos elementales como son: la empatía, el profesor debe tener la capacidad de ponerse en el lugar de su alumno,de esta manera la personalidad del educador ayudará significativamente al desarrollo de la competencia 


\section{LA EDUCACIÓN FÍSICA Y LAS RELACIONES SOCIALES EN EDUCACIÓN PRIMARIA}

social. La asertividad, se trata de una persona que está segura de sí misma, por lo tanto, un profesor que lleva a la práctica dicha asertividad puede facilitar la integración en el grupo, además de canalizar la agresividad y evitar conductas inadecuadas. La autoestima es el pensamiento crítico que tiene cada uno de sí mismo, en el aula se puede potenciar la autoestima a través de un ambiente de confianza, favorecer la iniciativa del escolar, tener expectativas realistas con los alumnos y permitir que los alumnos se expresen. La comunicación es muy importante entre los iguales porque de ese modo los alumnos tienen la oportunidad de decir todo lo que piensan, el profesor puede desarrollar esta técnica a partir del habla, de escuchar, escribir y leer (Otero, 2014).

\section{EL PAPEL DEL ALUMNO ANTE LAS RELACIONES SOCIALES.}

Según Ortega (1998) las relaciones interpersonales que se producen en el centro entre iguales son de gran importancia. Los alumnos tienen entidad como grupo, además de esta tendencia de agrupaciones, el grupo de iguales recibe desde fuera un tipo de retroalimentación que fortalece su identidad social de grupo.

Las interacciones que se producen en el contexto escolar con frecuencia se caracterizan por la ambigüedad de rol y por la coexistencia de relaciones positivas, neutras y negativas, con capacidad de producir efectos diferenciales sobre el comportamiento grupal (Huitsing et al., 2012).

Para los alumnos, todas las interacciones socialesles aportan una experiencia para aprender a ser más sociales, por lo tanto cada interacción que tengan va a influir en ellos de una forma muy directa en su vida y su desarrollo. Con estas actitudes interpersonales desarrollarán habilidades sociales, siempre que sean positivas, acrecentando sus comportamientos sociales.

Para el desarrollo de dichos comportamientos, los alumnos tienen que encontrarse un clima de confianza y afecto, ya que si ellos se sienten seguros podrán expresarse de una manera más libre. En un futuro, las consecuencias serán positivas, ellos podrán afrontar nuevos retos (Colmenares y Maldonado, 2006).

De la misma manera también influye la relación diaria entre profesor-alumno y alumno-alumno, ya que día a día se va desarrollando un clima de aceptación social, utilizando las habilidades sociales que tiene cada alumno, dichas habilidades son muy importantes ya que muchos estudios han afirmado que existe relación con el rendimiento, la creatividad y el propio desarrollo del comportamiento del alumnado (Colmenares y Maldonado, 2006).

\section{EXPRESIÓN CORPORAL}

Arteaga (2000) dice que el lenguaje se convierte en materia educativa y se utiliza para el desarrollo de la capacidad expresiva del ser humano a través de gestos, posturas y movimientos expresivos, fomentando el conocimiento personal, la comunicación interpersonal y la exteriorización de sentimientos.

Antolín (2013) define la Expresión Corporal como un acto consciente y voluntario que se desarrolla de forma intencionada con la participación del cuerpo. Se trata de una actividad humana que se puede analizar como un proceso donde intervienen los estímulos y la sensibilidad.

Una de las razones por integrar este bloque de contenidos es porque tiene la ventaja educativa de establecer relaciones sociales que fomentan la convivencia, puesto que se refuerzan los valores de respeto y la aceptación de producciones personales, además contribuye a aceptar la existencia de diferentes estímulos y a fomentar la indagación (Bardisa, 2005). 
Figura 2. Finalidades más importantes de la Expresión Corporal

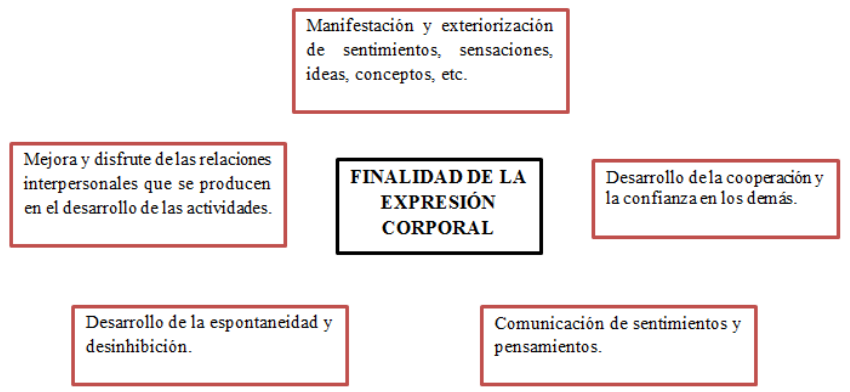

\section{EXPRESIÓN CORPORAL Y EDUCACIÓN FÍSICA}

La EC es una herramienta formativa que contribuye al desarrollo físico, intelectual y afectivoemocional de la persona (Vicente, Ureña, Gómez \& Carrillo, 2010).

Expresarse con el cuerpo es descubrir que tenemos algo que decir, y hay que aprender a decirlo con el lenguaje más apropiado, ya sea gesto, palabra, dibujo... No todo el mundo sabe hacerlo de la manera correcta, por eso la educación debe proporcionar al niño los medios necesarios para el descubrimiento y el conocimiento y con ellos su poder de comunicación (Fernández y Casanova, 2008).

Pujolás (2012) propone que a través del aprendizaje cooperativo se pueda llegar a alcanzar una educación inclusiva basada en sensibilizar al alumno a través de los valores de la inclusión, se trata de una educación adecuada a las características del alumnado, pudiendo trabajar de forma cooperativa y cada vez menos trabajo autónomo, potenciando el trabajo entre iguales. Como afirma Muntaner (2009) la cooperación favorece a la inclusión, por lo que el alumno participa y progresa.

Uno de los objetivos de la educación actual es la educación integral del alumno, por eso la Educación Física y por consiguiente el aprendizaje cooperativo junto con la Expresión Corporal aporta muchos beneficios y favorece el desarrollo de otros contenidos de forma conjunta y atractiva para el alumnado.

\section{EXPRESIÓN CORPORAL Y RELACIONES SOCIALES}

La EC tiene una gran relevancia en relación a la socialización del alumnado y el desarrollo de los sentimientos y emociones, donde la empatía tendría que ser el papel principal en estas actividades.

Está suficientemente demostrado que las personas que consiguen controlar su lenguaje corporal, son capaces de cruzar muchas barreras defensivas y establecer mejores relaciones sociales (Davis, 2010; Ekman, 2009; Pease y Pease, 2010), así como, a través de la empatía en actividades donde el alumno tenga que ponerse en el lugar de otra persona, para comprender y sentir qué emociones y sentimientos se despiertan en ellos, desarrollando una actitud empática con el compañero (Cascón, 2007).

En definitiva, la EC como materia educativa tiene implícita una serie de interacciones que posibilitan la comunicación entre los individuos participantes.

Una vez que la cooperación se asiente en las prácticas escolares como trabajo de centro educativo, será fundamental que el alumnado adopte ese papel y se establezcan relaciones simétricas con sus iguales en favor de una educación más rica, como apunta Pujolás (2012). 


\section{LA EDUCACIÓN FÍSICA Y LAS RELACIONES SOCIALES EN EDUCACIÓN PRIMARIA}

\section{DISEÑO DE LA UNIDAD DIDÁCTICA}

"Me expreso con mi cuerpo". Esta unidad didáctica se ubica concretamente en $2^{0}$ de primaria. La duración prevista se sitúa en torno a 3 semanas, aunque en función de la programación del centro, de los alumnos, etc. será el equipo docente quien realice las modificaciones oportunas.

\section{OBJETIVOS DIDÁCTICOS.}

Los objetivos propuestos serán los que se trabajarán en la unidad a través de las 7 sesiones planteadas.

Desarrollar las posibilidades expresivas de nuestro cuerpo.

Estructurar y contar los tiempos de la música en frases y estrofas, ajustando los movimientos,pasos básicos de danza y enlaces, a los distintos esquemas rítmicos.

Potenciar la autoestima abandonando la desinhibición.

Mejorar las relaciones interpersonales mediante la expresión y la danza, valorando la ayudade los compañeros/as en el desarrollo de las danzas.

Interpretar gestos y expresiones de los demás.

Desarrollar la cooperación, colaboración y la ayuda entre compañeros.

Desarrollar la capacidad de desinhibirse y la sensibilidad estética.

Desarrollar la no discriminación por razones de sexo, cultura, capacidad, religión.

\section{CONTENIDOS Y COMPETENCIAS BÁSICAS QUE SE DESARROLLAN.}

En esta unidad planteare una serie de contenidos que deben aprender los estudiantes y se trabajaran a lo largo de las sesiones programadas. Los alumnos deben ser capaces de alcanzar y superar todas las acciones propuestas, que además serán evaluadas a través de los criterios de evaluación.

Serán los siguientes: autoestima y desinhibición, relaciones interpersonales de cooperación, ayuda, colaboración y compañerismo, actitud crítica ante prejuicios sexistas, trabajos de expresión, de sensaciones, sentimientos y estados de ánimo, actividades de calidades del movimiento (pesado/ligero, fuerte/suave, rápido/lento etc.), juegos de improvisación de movimientos expresivos de forma individual y en grupos, juegos de representación de situaciones reales, expresividad, el cuerpo como instrumento de expresión y comunicación, recursos expresivos del cuerpo: el gesto y el movimiento ( mímica, danza y dramatización y estructuración de la música

En cuanto a las competencias, se trabaja la competencia de en el conocimiento y la interacción con el mundo físico, la competencia social y ciudadana y autonomía e iniciativa personal.

\section{METODOLOGÍA}

La metodología utilizada se compone de los diferentes estilos de enseñanza, para conseguir a través de ellos un aprendizaje cooperativo en los alumnos.

Todos los estilos trabajados en las sesiones priorizan la cooperación y colaboración frente a la competición. Por ello, sobre todo, a través de los estilos de socialización y expresión libre se fomenta la cooperación. Se trata de un método donde los alumnos reciben consignas de actuación por parte del profesor, y a partir de ahí, son ellos mismos los responsables de organizar y planificar cómo lo quieren hacer.

Con los grupos formados, se pretende buscar la máxima diversidad basándonos en la Educación por la igualdad de sexos como tema transversal, ya que el aprendizaje de cooperación e interacción social se alcanza mejor afrontando la diferencia trabajando la educación moral y cívica así como la educación para la paz.

La elección de escoger este método de aprendizaje para esta propuesta, es porque el resulta- 
dodel estudio de Avcioglu (2004), afirma que las habilidades sociales como un programa basado en el método de aprendizaje cooperativo, la conclusión del estudio fue que a través del programa de enseñanza de habilidades sociales desarrolladas en la dirección del método de aprendizaje cooperativo, se encontró que tienen un efecto positivo de las habilidades sociales en los estudiantes con las cuales se desarrolla la comunicación (citado por Görgülü, 2009).

Otra razón por optar por el AC como método es porque según Gillies (2006) actualmente es considerado una importante estrategia de enseñanza-aprendizaje, que promueve logros a nivel académico, social y afectivo-emocional en todos los estudiantes. Asimismo, el alumnado aumenta el grado de implicación en las tareas, se potencian las habilidades sociales, habilidades de comunicación oral y escrita, afectando favorablemente a los niveles de comprensión de textos (Fernández-Río, 2003; Velázquez Callado, 2006, 2010).

\section{SESIONES}

Sesión 1: "Representamos animales"

Sesión 2: "Representación e imitación de gestos y emociones"

Sesión 3: "Simulaciones y representaciones"

Sesión 4: "Iniciación al ritmo"

Sesión 5: "El ritmo"

Sesión 6: "Danza"

Sesión 7: "¡A bailar!"

El primer día de práctica, se utilizará para ver las relaciones entre los alumnos y sus diferencias.

La segunda semana, desarrollarán diferentes actividades donde se inicien en las habilidades artístico-expresivas.

Para finalizar, en la última sesión realizaremos las sesiones correspondientes a la puesta en práctica de una coreografía.

\section{MODALIDADES, MOMENTOS E INSTRUMENTOS DE EVALUACIÓN.}

La evaluación va a tener un carácter continuo, utilizando como procedimiento de evaluación la observación directa. Según Puente (2009) la observación directa es una técnica que consiste en observar atentamente el fenómeno, hecho o caso, sin intervención, con el fin de tomar información y registrarla para su posterior análisis. La observación es un elemento fundamental de todo proceso investigativo; en ella se apoya el investigador para obtener el mayor número de datos. Gran parte del acervo de conocimientos que constituye la ciencia ha sido lograda mediante la observación.

En la evaluación se pueden diferenciar tres momentos pese a ser un proceso continuo: Evaluación inicial: se realiza al comienzo para ver el nivel de los alumnos, se usa la observación directa para ver los diferentes comportamientos entre ellos, así de una manera u otra poder enfocar las restantes sesiones. Evaluación continua: durante el proceso de enseñanza-aprendizaje, se realizará un registro anecdótico, en el cual se irá apuntando los aspectos que más destacan de las sesiones que nos puede servir para remodelar alguna sesión posterior para corregir posibles errores de planteamiento en el proceso de enseñanza.Evaluación final: se realizará al final de la unidad didáctica para valorar el grado de adquisición de los contenidos por parte de los alumnos. Para ella utilizaremos una lista de control en la cual valoraremos la consecución o no de estos.

Como instrumentos de evaluación se ha utilizado la lista de control y el cuaderno de campo, donde se recopilará un registro narrativo. A través de ello se obtendrá información relevante sobre lo realizado, alcanzado y superado. 


\section{LA EDUCACIÓN FÍSICA Y LAS RELACIONES SOCIALES EN EDUCACIÓN PRIMARIA}

\section{CONCLUSIONES Y FUTURAS LÍNEAS DE INTERVENCIÓN}

A lo largo del trabajo se han mencionado algunos aspectos relevantes para poder entender la finalidad de este. La hipótesis de trabajo era si el área de Educación Física permitía el desarrollo de las relaciones sociales a través de la Expresión Corporal aplicando el aprendizaje cooperativo, en un primer momento, se da por supuesto que la Educación Física desarrolla la interrelación entre los alumnos. No obstante, no está tan claro que la Expresión Corporal pueda hacerlo, asimismo, cabe destacar que no todos los maestros ponen en práctica estos contenidos, o bien por la falta de información sobre dicho tema o por el miedo a no desarrollarlo de una manera correcta, y en mi opinión, es algo que tendría que estar dentro de la normalidad y del día a día del docente.

La Expresión Corporal como contenido promueve las habilidades sociales gracias al aprendizaje cooperativo en Educación Primaria. A lo largo de todo el trabajo se pueden observar todos los beneficios que genera la Expresión Corporal, en general contribuye al bienestar personal de los alumnos (Cuellar, 2004).

Por lo tanto, con los datos reunidos, se podría decir que gracias a la Expresión Corporal trabajada desde el aprendizaje cooperativo, se afianzan las relaciones sociales. Sin embargo, habría que llevar la propuesta didáctica a la práctica real para poder verificarlo completamente.

El objetivo principal (Investigar la relación existente entre la Educación Física y la consolidación de las relaciones sociales a través de un aprendizaje cooperativo con una propuesta de intervención de Expresión Corporal, en los alumnos de Educación Primaria) se cumple de tal manera que gracias a esta materia se puede fomentar y desarrollar un trabajo cooperativo en todas las actividades propuestas, y así mejorar las relaciones sociales entre los alumnos.

También, creo necesario hacer una valoración de la Expresión Corporal, con lo que conlleva (bailes, movimientos, simulaciones, contacto personas, etc.), como una actividad importante para el desarrollo integral del niño (Grasso, 2006), esta actividad favorece el desarrollo integral del alumno en la etapa de primaria, porque al llevarla a cabo los alumnos están saltando, girando, imitando, etc. es decir, están desarrollando sus capacidades perceptivo-motrices, donde también desarrollan la expresividad, la creatividad y por supuesto la imaginación al expresar lo que sienten, dejándose llevar por sus compañeros o por la música.

Al trabajar actividades de forma grupal se produce una mayor socialización entre los integrantes del grupo y el resto de los compañeros, a la vez que el niño va cogiendo confianza en sí mismo y eliminando la sensación de ridículo que pueda sentir al expresar lo que siente con su cuerpo, ya que se trata de algo que no hacen nunca o casi nunca.

Otra conclusión importante, es que el niño de forma lúdica aprende a conocer su cuerpo, sintiéndose de una manera libre desplazándose por el gimnasio, teniendo en cuenta el tiempo y el espacio, así como las diferentes canciones que escuchan, de esta manera el alumno toma conciencia de la necesidad de conocer su cuerpo y el de los demás, respetando las visibles diferencias que puedan tener, por lo tanto es otra razón por optar por la Expresión Corporal como fomento de las relaciones sociales entre ellos.

\section{REFERENCIAS}

Antolín, L. (2013). Expresión Corporal Fundamentos Motrices. Valencia: Universitat de València.

Brigido, A. M. (2006). Sociología de la Educación. Recuperado de http://www.docsengine.com/pdf/1/ana-maria-brigido-sociologia-de-la-educacion-temas-y-perspectvas-fiundamentales.html

Camacho, Á. S., y Noguera, M. Á. D. (2002). Educación física y estilos de enseñanza: análisis de la participación del alumnado desde un modelo socio-cultural del conocimiento escolar. Madrid: Inde. 
Departamento de Educación, Cultura y Deporte. (2014). RD 1262014 Currículo Básico Educación Primaria. Recuperado de http://www.educaragon.org/HTML/carga_html.asp?id_submenu=52

Dos Santos, S. J. (2015). Association between physical activity, participation in Physical Education classes, and social isolation in adolescents. Jornal de Pediatria, 91(6), 543-550.

Frank, M. A. (2011). The Pillars of the Self-Concept: Self-Esteem and Self-Efficacy. Recuperado de https://www.excelatlife.com/articles/selfesteem.htm

Frank, M. A. (2011). The Pillars of the Self-Concept: Self-Esteem and Self-Efficacy. Recuperado de https://www.excelatlife.com/articles/selfesteem.htm

Sánchez, I. G., Ordás, R. P. y Lluch, Á. C. (2013). Expresión corporal. Una práctica de intervención que permite encontrar un lenguaje propio mediante el estudio y la profundización del empleo del cuerpo. Nuevas tendencias en Educación Física, Deporte y Recreación, 23, 19-22.

Crespo, M. A. G. (2006). Aprendizaje e instrucción en Química. El cambio de las representaciones de los estudiantes sobre la materia. Madrid: C.I.D.E

Gallegos, A. G., Extremera, A. B., López, M. G., y Abraldes, J. A. (2014). Importance of Physical Education: motivation and motivational climate. Procedia-Social and Behavioral Sciences, 132, 364-370.

Grasten, A. (2015). Children's expectancy beliefs and subjective task values through two years of school-based program and associated links to physical education enjoyment and physical activity. Journal of Sport and Health Science, 4, 10.

Güleç, S. (2013). The Effects of Drama-supported Cooperative Learning Method on the Development of Pre-school Children's Communication Skills. Procedia - Social and BehavioralSciences, 136, 532-536.

Hernández, A., Martínez, F. D. y Sánchez, V. M. (2011). Construcción de una herramienta observacional para evaluar las conductas prosociales en las clases de educación física. Psicología del deporte, 19, 305-318.

Jurado, J. (2015). Cuerpo de maestros (Música), Madrid: Primera Edición 7 editores, MAD.

Lagunas, J. M. (2006). Educación física y desarrollo integral. Recuperado de file:///C:/Users/Tania/Downloads/Dialnet-EducacionFisicaYDesarrollolntegral$2543127 \% 20(3)$.pdf

Learreta, B., Sierra, M. A. y Arriagada, K. R (2005). Los contenidos de Expresión Corporal. Barcelona: INDE publicaciones.

Leea, H., Parsonsb, D., Kwona, G., Kima, J., Petrovac, K., Jeonga, E. y Ryua, H. (2016). Cooperation begins: Encouraging critical thinking skills through cooperative reciprocity using a mobile learning game. Computers\&Education, 97, 97-115.

Zarceño, E. M. L. (2008). Motivación de conductas prosociales en la escuela a través del deporte. Valencia: Universitat de València.

Lorenzana, L. (2012). Conducta humana, motivación, rasgos de personalidad y los tipos de forma de ser. Superación personal. Psicología motivacional. Recuperado de http://biblio3.url.edu.gt/Tesario/2014/05/42/Mazariegos-Ingrid.pdf

Maxwell, L. E. (2016). School building condition, social climate, student attendance and academic achievement: A mediation model. Journal of EnvironmentalPsychology, 46, 206-216.

Muñoz, L. F. M. (2004). Los significados de la motricidad en el recreo como caleidoscopio de la educación del ocio en la escuela. Valladolid: Universidad de Almería.

Marashian, F. y Khorami, N. S. (2012). The Effect of Early Morning Physical Exercises on Academic Self-concept and Loneliness Foster Home Children in Ahvaz City. Procedia - Social and BehavioralSciences, 46, 316-319. 


\section{LA EDUCACIÓN FÍSICA Y LAS RELACIONES SOCIALES EN EDUCACIÓN PRIMARIA}

Otero, V. M. (2007). La buena educación. Reflexiones y propuestas de psicopedagogía humanista. Barcelona: Anthropos Editorial.

Otero, V. M. (2014). Competencia social en la Escuela. Recuperado de http://www.psicopedagogia.com/articulos/?articulo=393

Molina de Colmenares, N. y Pérez de Maldonado, I. (2006). El clima de relaciones interpersonales en el aula un caso de estudio. Paradígma, 27(2), 193-219.

Mustafa Er. (2014). Procedia - Social and Behavioral Sciences. Procedia - Social and Behavioral Sciences, 69, 535-544.

Nadal, C. B., Soler, V. G. y García, P. D. (2014). La importancia de las competencias emocionales en la gestión de unidades organizativas. 3Ciencias, 3 (1), 42-61.

Pereira, M. L. N. (2009). Motivación: perspectivas teóricas y algunas consideraciones de su importancia en el ámbito educativo. Revista de Educación, 33 (2), 153-170.

Nichele de Chavesa, R. (2016). Developmental and physical-fitness associations with gross motor coordination problems in Peruvian children. Research in DevelopmentalDisabilities, 53-54, 107-114.

Pujolás, P. (2009). 9 ideas clave. Aprendizaje cooperativo. Barcelona: Editorial GRAÓ.

Ortega, R. (2000). La convivencia escolar: qué es y cómo abordarla. Recuperado de http://harcelement-entreeleves.com/images/presse/convivenciaqosarioortega.pdf

Sánchez, R. B., Mallado, C. M. y González, R. P. (2013). Cambios pedagógicos y sociales en el uso de las TIC. Departamento de Pedagogía, 10, págs. 7-20.

Sánchez-Oliva, D. (2015). Análisis de los perfiles motivacionales y su relación con los comportamientos adaptativos en las clases de educación física. RevistaLatinoamericana de Psicología, 47 (3), 156-166.

Sherrill, C. (2004). Adapted physical activity, recreation and sport: Crossdisciplinary and lifespan(6th ed.). Boston, MA: McGraw-Hill Higher Education, 736p.

Tandon, P. S. (2016). The relationship between physical activity and diet and young children's cognitive development: A systematic review. Preventive Medicine Reports, 3, 379-390.

Tilli, P. (2012). Apreciación Didáctica. Mr. Holland's Opus. Buenos Aires: ACADEMIA

Orts, J. V. (2005). Las habilidades sociales en el aula. Madrid: Santillana.

Velázquez Callado, C. (2015). Aprendizaje cooperativo en Educación Física: estado de la cuestión y propuesta de intervención. Federación Española de Asociaciones de Docentes de Educación Física (FEADEF), 28, 234-239.

Velea, S. (2013). Teacher's Responsibility in Moral and Affective Education of Children. Procedia. Social and Behavioral Sciences, 76, 863-867.

Velea, S. y Farca, S. (2013). Teacher's Responsibility in Moral and Affective Education of Children. Procedia - Social and BehavioralSciences, 76, 863-867.

Vidal, I. R. (2015). Popularidad y relaciones entre iguales en el aula: un estudio prospectivo. PsicologíaEvolutiva, 1.

Wadman, K. Durkin, G. y Ramsden, C. (2011). Social stress in young people with specific language impairment. Journal of Adolescence, 34 (3), 421-431. 